\title{
Observing strain localisation processes in bio-cemented sand using x-ray imaging
}

\author{
Francesco Tagliaferri - Jack Waller • Edward Andò - Stephen A. Hall · \\ Gioacchino Viggiani · Pierre Bésuelle · Jason T. DeJong
}

Received: date / Accepted: date

\begin{abstract}
In-situ x-ray tomography has been used to follow deformation processes in 3D during two triaxial compression tests, one on a specimen of bio-cemented Ottawa 50-70 sand and the other on a specimen of the non-cemented sand. The global stress-strain responses show that the bio-cementation process increases the shear strength (peak deviator stress is approximately doubled), and causes the material to exhibit a linear behaviour up until peak, as well as increasing the dilatancy angle. The residual strength of the two samples is very close at large strain. Quantitative $3 \mathrm{D}$ digital image analysis (porosity, cement-density and strain field measurements), reveals that a dilatant shear band gradually develops pre-peak in the reference material. The cemented sample however undergoes an abrupt change of deformation mechanism at peak stress: from homogeneous deformation to localised dilatant shearing, which is associated with a local loss of cementation.
\end{abstract}

Keywords bio-cemented sand · strain localisation . In-situ x-ray tomography $\cdot 3 \mathrm{D}-\mathrm{DIC}$

\section{Introduction}

Bio-cemented soil improvement is an environmentally benign alternative to grouting techniques traditionally used to stabilise sand. The technique uses Bacillus Pasteurii, a subsurface bacterium, to cement sand grains

Francesco Tagliaferri · Edward Andò . Stephen A. Hall . Gioacchino Viggiani · Pierre Bésuelle

CNRS / UJF / G-INP, Laboratoire 3SR, Grenoble, France

E-mail: cino.viggiani@hmg.inpg.fr

Jack Waller · Jason T. DeJong

Department of Civil and Environmental Engineering, Univ. of California, Davis, CA, USA

E-mail: jdejong@ucdavis.edu together by means of calcite precipitation (e.g., [1]). Whilst studies have been made of the change in macroscopic sand behaviour after bio-cementation (e.g., [2]), little is known about alteration of deformation mechanisms at the grain scale, which is important in the development of accurate models.

Previous work has demonstrated how in-situ x-ray microtomography (in-situ meaning x-ray scanning during loading) combined with analysis of the resulting images can improve understanding of grain-scale deformation mechanisms and, in particular, the processes accompanying strain localisation (e.g., [3]). In this current work the alteration of the mechanics of sand due to biocementation is investigated. This is done at the grainscale using image analysis to follow processes in $3 \mathrm{D}$ during triaxial compression tests on reference and biocemented sand. 3D tomography images are analysed using quantitative 3D (volumetric) digital image analysis, including 3D porosity and cement-density analysis and $3 \mathrm{D}$ strain tensor measurements by 3D-volumetric Digital Image Correlation (DIC). The experimental methods (sample preparation, in-situ triaxial tests and subsequent 3D image analysis) are first presented followed by a comparison of two triaxial compression tests, one on a cemented sample of Ottawa sand and one on a reference sample of the same material.

Ioannis Vardoulakis never worked on bio-cementation, but he might well have done. In fact, he was - among many other things - an innovative experimentalist. He used x-rays already in the 1970 s and he realised the great potential of tomography for studying shear banding in granular materials. Cino Viggiani recalls, at a workshop in 1993 (in Aussois), Ioannis taking the floor after seeing Jacques Desrues' results of x-ray tomography on sand[4] and, in his charismatic way, announcing to the audience that this tool was destined to revo- 
lutionise research in geomechanics. We hope that this work will, at least in part, help fulfill this prophecy.

\section{Experimental methods}

The material studied in this work is the Ottawa 50-70 sand. This is a quartz sand with well-rounded grains and an average diameter $\left(D_{50}\right)$ of approximately 250 $\mu \mathrm{m}$; the granulometry is very narrow, with $97 \%$ of the grains having diameters between 212 and $300 \mu \mathrm{m}$. The cemented specimen was prepared by pluviating sand into a cylindrical mould (11 $\mathrm{mm}$ diameter and 22 $\mathrm{mm}$ height), and then bio-cementing by precipitation of calcite (facilitated by bacteria); see [2]. Any desired level of cementation can be achieved by repeating this treatment process a number of times: in this work 30 treatments were used, alternating the direction of fluid pumping between treatments. The reference sample was prepared by pluviation into a mould of the same dimensions. Figure 1 shows a comparison of the two materials as imaged by x-ray tomography. Note that the image of the cemented specimen was acquired at a greater spatial resolution than the reference sand to provide detail on the cement distribution. However, the images from the in-situ test are of approximately the same resolution.

During the triaxial compression tests, x-ray tomography scans made at different strain levels using the x-ray tomograph at 3SR (Grenoble). Axial loading was pasued in order to scan with a fixed piston position (note that, as in previous work, e.g., [3], an initial, almost instantaneous, relaxation was observed after loading was halted - see Figure 2a). The resulting 3D images have spatial resolution of about $15 \mu \mathrm{m}$, which allows individual grains to be clearly identified (recall that $D_{50}$ is about $250 \mu \mathrm{m}$, giving a few thousand voxels per grain).

3D porosity maps (Figures 3a, 4a) were derived from thresholded images of the pore and solid phases. Threshold values were chosen to include the number of voxels corresponding to the volume of the solid fraction. This is calculated post-test from the measured grain mass (after the cement had been dissolved away by acid) and known density of quartz. The porosity field was calculated in sub-volumes of 60x60x60 voxels (the smallest volume giving spatially stable measurements of porosity) over the whole sample over a 3D mesh with a 20 voxel grid spacing. Likewise, cement distributions (Figure 3c) were calculated using a thresholded image of cement and non-cement (i.e., grains and pores); in this case the threshold value was chosen to correspond to the measured cement volume.

Strain fields, and their evolution during loading, have been quantified from 3D-volumetric DIC (TOMOWARP code; e.g., [3], [5]). DIC compares two 3D images of a
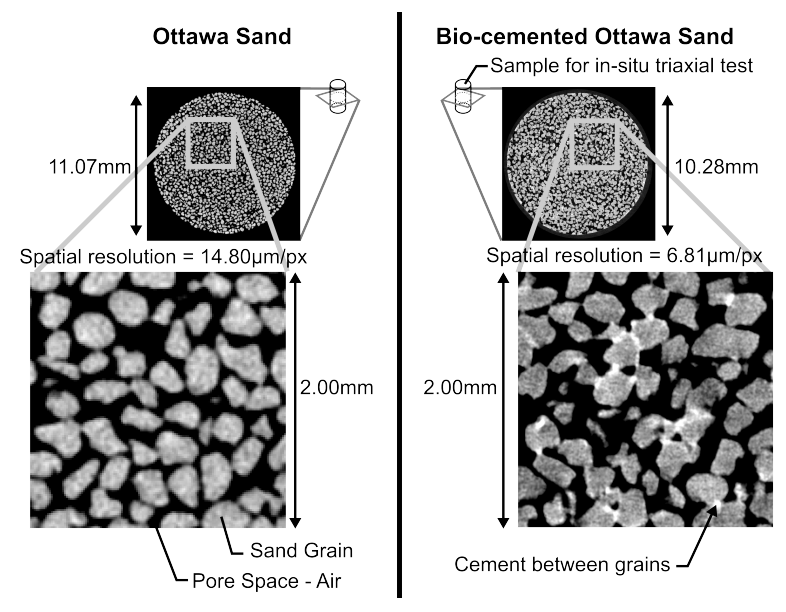

Fig. 1 Horizontal slices through 3D x-ray tomography images of the cemented and reference sand specimens indicating the geometry and organisation of the grains plus the distribution of cements in the treated specimen (the cement appears brighter than the quartz grains due to the higher density of calcite).

specimen (a reference and a deformed image) over a regularly-sampled, 3D grid by searching for the 3D displacement that gives the best correspondence between small subvolumes about each point of the grid. This mapping requires a number of steps including sub-voxel refinement, as described in [3]. The output from DIC, which is applied here to consecutive pairs of $3 \mathrm{D}$ images of the samples throughout the test, are 3D maps of incremental displacement vectors, from which tensorial strain fields can be derived.

\section{Results: Macroscopic response}

Results are presented here from two triaxial compression tests at $100 \mathrm{kPa}$ confinement, one on the biocemented sand and one on the reference sand; the samples were tested dry and had initial relative densities of $74 \%$ and $84 \%$, respectively. The higher relative density of the cemented sample is a direct result of cementation, since the two specimens start from approximately the same initial porosity. Loading was carried out under displacement control at a constant axial displacement rate of $21 \mu \mathrm{m} / \mathrm{min}$. Figure 2a shows the stress-strain response of the two specimens.

The mechanical effect of cementation manifests itself principally before the peak stress; the peak is higher (shear strength increased by a factor of 1.8 ), occurs at lower axial strain (1.5\% rather than $7 \%$ ) and follows a linear stress-strain response. The deviator stress at large strain appears to be tending to a similar (ultimate) value in the two tests. Figure $2 \mathrm{~b}$ shows the volumetric strain of the two samples (calculated from the 


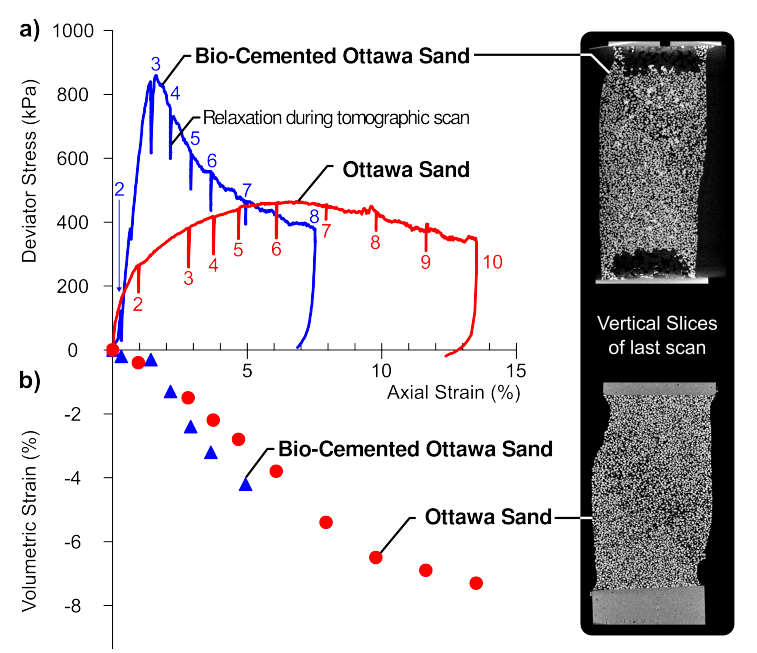

Fig. 2 Macroscopic sample responses. (a) Deviatoric stress vs. axial strain for the two triaxial compression tests discussed. The labelled relaxations correspond to x-ray scans. (b) Volumetric strain vs. axial strain calculated from tomography images.

$\mathrm{x}$-ray tomography images) as a function of the axial strain. The reference sample can be seen to dilate from the start of deviatoric loading whilst the cemented specimen starts to dilate after about $1.5 \%$ axial strain i.e., just before the peak stress is attained, and does so at an initially higher dilatancy angle.

\section{Results: full-field measurements}

Figures 3 and 4 show, for both tests, a selection of vertical slices through the calculated porosity volumes and the incremental shear strain fields at different stages of loading. In addition, the corresponding slices through the calculated cement density volume for the cemented sample are presented in Figure 3c. The slices are roughly orthogonal to the shear band that forms with loading.

For the cemented specimen, the porosity fields (Figure 3a) indicate that the sample was initially reasonably homogeneous and no clear change occurred until well after the peak stress (around scan 5). After this moment a band of localised dilation is seen inclined at an angle of $50^{\circ}$. This band corresponds to a localised zone of elevated incremental shear strain (Figure $3 \mathrm{~b}$ ) that is evident in the loading increment $3-4$, which traverses the loading peak, and indications may exist in increment 23 , i.e., before the peak. The sample can be considered to be initially fully cemented. The cement distribution is axially-homogeneous (thanks to the reversal of the flow direction), but radially-concentrated in the middle of the sample (due to inadequate radial diffusion); note that some cement thickness may be below the image resolution, and therefore may go undetected. Dur-

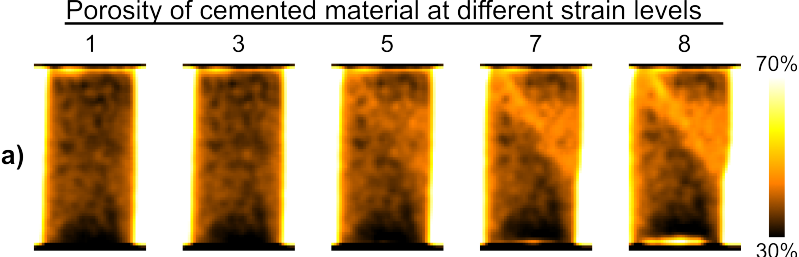

Incremental shear strain between strain levels

$\begin{array}{lllll}2 \Rightarrow 3 & 3 \Rightarrow 4 & 4 \Rightarrow 5 & 6 \Rightarrow 7 & 7 \Rightarrow 8\end{array}$

b)

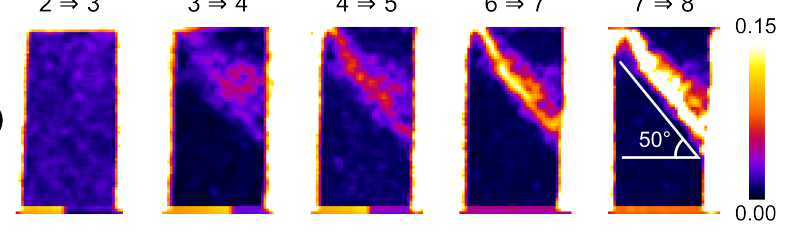

$\frac{\text { Cement distribution at different strain levels }}{3}$

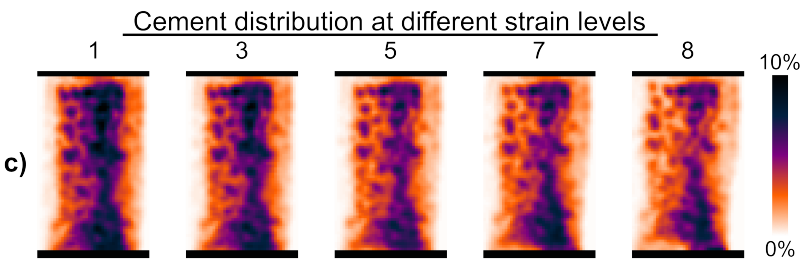

Fig. 3 Cemented specimen image analysis results — slices (oriented orthogonal to shear band) through 3D maps of: (a) porosity; (b) DIC-derived shear strain; (c) cement density evolution (for selected key load steps).

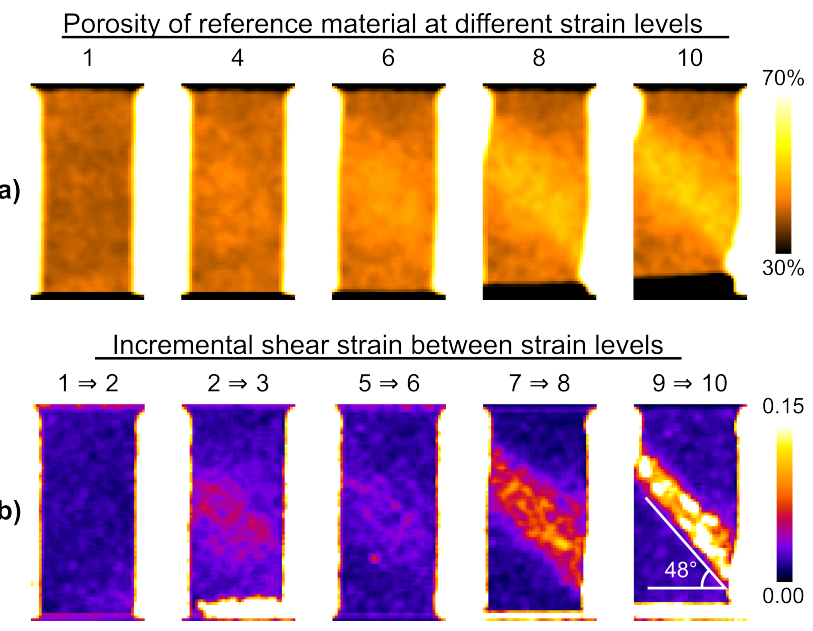

Fig. 4 Reference specimen full-field results (as Figure 3a,b).

ing loading, the cement distribution decreases in the zone where the shear band develops (Figure 3c), which might be explained by the hypothesis that, as cement bonds break, the cement is broken into particles below the resolution of the tomography such that the cement effectively "disappears" from view.

The porosity fields for the reference sample also indicate a good initial homogeneity. A central zone of dilation appears well before the peak (this is evident at step 6, indications can be seen from step 4). This dilation zone develops into a large, but well localised, 
dilation band by the end of the test traversing the sample at $48^{\circ}$. The strain fields tell a similar story, but again reveal the localisation at an earlier stage than the porosity fields. In increment 5-6 there is a reasonably well developed localised shear band traversing the sample, which had already started in increment 2-3. The incremental shear strain fields show a much more localised feature by the end of the test than is revealed by the porosity (the latter being an accumulated, i.e., total, measure over all the preceding increments).

\section{Discussion and conclusions}

The macroscopic response of the reference sand is typical of dense sand, with dilatancy throughout the test. In fact, the full-field measurements of porosity show that when the shear band forms the dilatancy is concentrated within it. The images of shear strain highlight large levels of localised shear strain occurring in the band, which starts wide and condenses into an active band between 12 and 13 grain diameters wide. Both measurements show the development of localised phenomena before the peak.

Macroscopically, the effect of the cement is clear: increasing the shear strength of the material by a factor of 1.8 and causing the material to behave linearly up to the peak. After the peak, there is considerable softening, eventually recovering the same residual strength as the reference sand. These "macroscopic" observations are in accordance with previous work (e.g., [2]), despite the reduced sample size. The full-field measurements offer some insight into the micromechanisms of deformation: up to the stress peak, the shear strain field appears to be homogeneous, as do the (small) changes in the porosity and cement distributions.. The presence of cement bonds between grains appears to allow the material to resist the onset of localisation. Since the cement offers a one-off bond between the grains, this additional strength is lost when the cement bonds break. Cement distribution maps confirm this showing a reduction in the degree of cementation in the region of localised deformation. The material is thus locally weakened and the strength returns to that of the non-cemented sand, causing additional shear strain to be concentrated in this weakened zone. This explains why the shear band is tighter when it develops in the cemented sample, visible both in porosity and shear strain measurements.

The cemented material appears to have two mechanisms of deformation: (1) homogeneous, linear initial response, due to the cement bonding the grains together, resisting localised deformation; (2) concentrated, localised deformations where the cement is lost locally and the residual strength of the sand is eventually recovered in the band. Transition from mechanism 1 to 2 is abrupt and occurs around the peak, which might be explained by the bonds reaching their brittle failure strength, causing a local weakness that attracts stress and triggers further localisation.

The angle of the shear band is not strongly affected by the cementation process. Furthermore, at large strains both samples reach the same residual strength, indicating that the two materials have similar friction angles. The higher stress peak can therefore be interpreted as being due to both cohesion and dilatancy (see Figure $2 \mathrm{~b})$. After the peak, the cohesive bonds in the shear band are observed to be broken, and the difference in strength between the two samples only comes from different mobilised dilatancies.

It should be stressed that the results presented herein are preliminary. Nevertheless they indicate some of the microscale origins of the differences in mechanical behaviour after bio-cementation. Further tests are to be carried out to complement these results and investigate different factors such as the level of cementation.

\section{Acknowledgements}

We thank Ioannis for all his great contributions to our discipline over the years.

We acknowledge the help of Pascal Charrier (3SR) in the experimental work. Funding was provided for 3SR by the French ANR (project GeoBridge at 3SR) and for UCD by the United States National Science Foundation (\#0727463). Any opinions, findings, and conclusions or recommendations expressed in this material are those of the writers and do not necessarily reflect the views of the National Science Foundation.

\section{References}

1. DeJong, J.T., Mortensen, B. M., Martinez, B. C., Nelson, D.C.: Bio-mediated soil improvement. Ecological Engng., 36, 197-210 (2010)

2. DeJong, J.T., Fritzges, M.B., Nüsslein K.: Microbially induced cementation to control sand response to undrained shear. J. Geotech. and Geoenv. Engng., 132, 1381-1392 (2006)

3. Hall, S.A., Bornert, M., Desrues, J., Pannier, Y., Lenoir, N., Viggiani, G., Bésuelle, P.: Discrete and continuum experimental study of localised deformation in Hostun sand under triaxial compression using X-ray $\mu \mathrm{CT}$ and $3 \mathrm{D}$ digital image correlation. Géotechnique, 60, 315-322 (2010)

4. Desrues, J., Chambon, R., Mokni, M., Mazerolle, F.: Void ratio evolution inside shear bands in triaxial sand specimens studied by computed tomography. Géotechnique, 46, 529-546 (1996)

5. Viggiani, G., Hall, S. A.: Full-field measurements, a new tool for laboratory experimental geomechanics. Keynote paper. Proc. 4th Int. Symp. Deformation Characteristics Geomaterials (eds S. E. Burns et al.), Atlanta: IOS Press, Vol. 1, 3-26 (2008) 